\title{
EVOLUÇÃO DA PAISAGEM E DINÂMICA SEDIMENTAR DO RIO TEJO EM PORTUGAL, DURANTE O PLISTOCÉNICO - REGISTO EM PERFIS LONGITUDINAIS REGULARIZADOS COM SUBSTRATO ROCHOSO E NOS DE LEITO ALUVIAL
}

\author{
António A. Martins ${ }^{(a)}$, Pedro P. Cunha ${ }^{(b)}$ \\ (a) Instituto de Ciências da Terra; Departamento de Geociências, Universidade de Évora, aam@uevora.pt \\ (b) MARE - Centro de Ciências do Mar e do Ambiente; Departamento de Ciências da Terra da Universidade de \\ Coimbra; pcunha@dct.uc.pt
}

\section{Eixo: GEOCRONOLOGIA E ESTUDOS PALEOAMBIENTAIS}

\begin{abstract}
Resumo
Em Portugal, o Rio Tejo desenvolveu seis níveis terraços fluviais desde a superfície culminante do enchimento sedimentar da Bacia Cenozóica do Baixo Tejo até ao leito actual. A unidade culminante, com idade de 3,7 a 1,8 Ma, e que se encontra a ca. +142 a $262 \mathrm{~m}$ acima do leito do Tejo, representa ainda um ancestral Tejo, antes do início da etapa de incisão fluvial, durante a qual se desenvolveram os terraços fluviais, enquadrados por vertentes. $\mathrm{O}$ terraço $\mathrm{T} 1 \mathrm{e}$ correspondente lateral superfície de erosão N1 (+84 a 180 m; ca. 1Ma a 900 ka), parecem representar um longo período de estabilidade da rede fluvial (steady state) anterior ao Plistocénico Médio. A formação desta unidade morfoestratigráfica deverá ter correspondência com troços "relíquia" regularizados (graded profiles) com substrato rochoso e concavidade elevada, situados nas cabeceiras dos tributários do Tejo. O terraço T2 (+57 a $150 \mathrm{~m})$, com idade estimada em ca. $600 \mathrm{ka}$, deverá corresponder à fase final da "Revolução do Plistocénico Médio". Esta unidade está mal representada, provavelmente por ser relativamente antiga e porque o tempo desenvolvimento foi menor que o do terraço T1. Os terraços mais baixos T3, T4, T5 e T6 testemunham o progressivo estreitamento do vale e formaram-se durante o Plistocénico Médio e Final, períodos caracterizados por oscilações climáticas de grande amplitude e intensificação do soerguimento regional. $\mathrm{Na}$ área de estudo, para o último milhão de anos, as taxas de incisão fluvial poderão variar, entre 0,38 e $0,12 \mathrm{~m} / \mathrm{mil}$ anos, dependendo do soerguimento diferencial entre compartimentos limitados por falhas de importância regional.
\end{abstract}

Palavras chave: terraços fluviais; perfis longitudinais, nickpoints, soerguimento tectónico, rio Tejo

\section{Introdução}

Nas áreas mais interiores de Portugal, o Rio Tejo corre no soco arrasado do Maciço Hespérico (Meseta Ibérica), situada na área de estudo aos 300 a 400 m acima do nível do mar. Esta superfície de erosão corta rochas metassedimentares e granitóides de idade Neoproterozoica e Paleozoica. O Tejo cruza esta superfície num vale encaixado cerca de $250 \mathrm{~m}$, mas desprovido de terraços (Fig. 1). Na grande Bacia Cenozóica do Baixo Tejo o rio desenvolve uma escadaria de seis terraços a partir da superfície fluvial culminante (SFC) da bacia cenozóica. Na área mais interior de Portugal, o Tejo recebe tributários da superfície da Meseta, mas também da Cordilheira Central Portuguesa (CCP), ex. Rib. da Sertã e Rib. da 


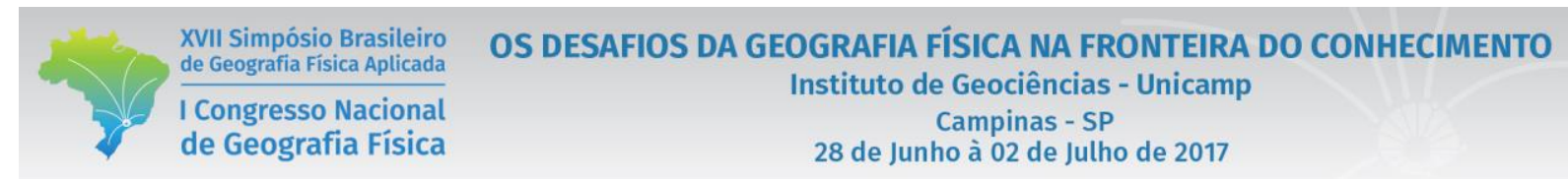

Isna, (Fig.1). Nestas áreas interiores do território, a utilização de superfícies de erosão e superfícies agradacionais de idade imprecisa tem sido utilizada para o cálculo do soerguimento regional (Cabral, 2012). Na mesma área, os tributários do Tejo, quase sempre desprovidos de terraços sedimentares, apresentam um troço a montante regularizado (graded profile) e pouco encaixado em contraste com o troço a jusante, rejuvenescido e parcialmente regularizado. Esta particularidade é reconhecível em alguns tributários do Tejo, no interior CCP, mas é muito frequente nos afluentes provenientes da superfície da Meseta. Nestes afluentes, o perfil longitudinal do leito rochoso do rio principal e dos tributários é, muitas vezes, a única informação geomorfológica disponível para conhecer a evolução de longo prazo da paisagem destes sectores, pois os registos dos terraços encontram-se espacial e temporalmente, fragmentados e, quando preservados, estão quase sempre associados ao sistema fluvial principal.

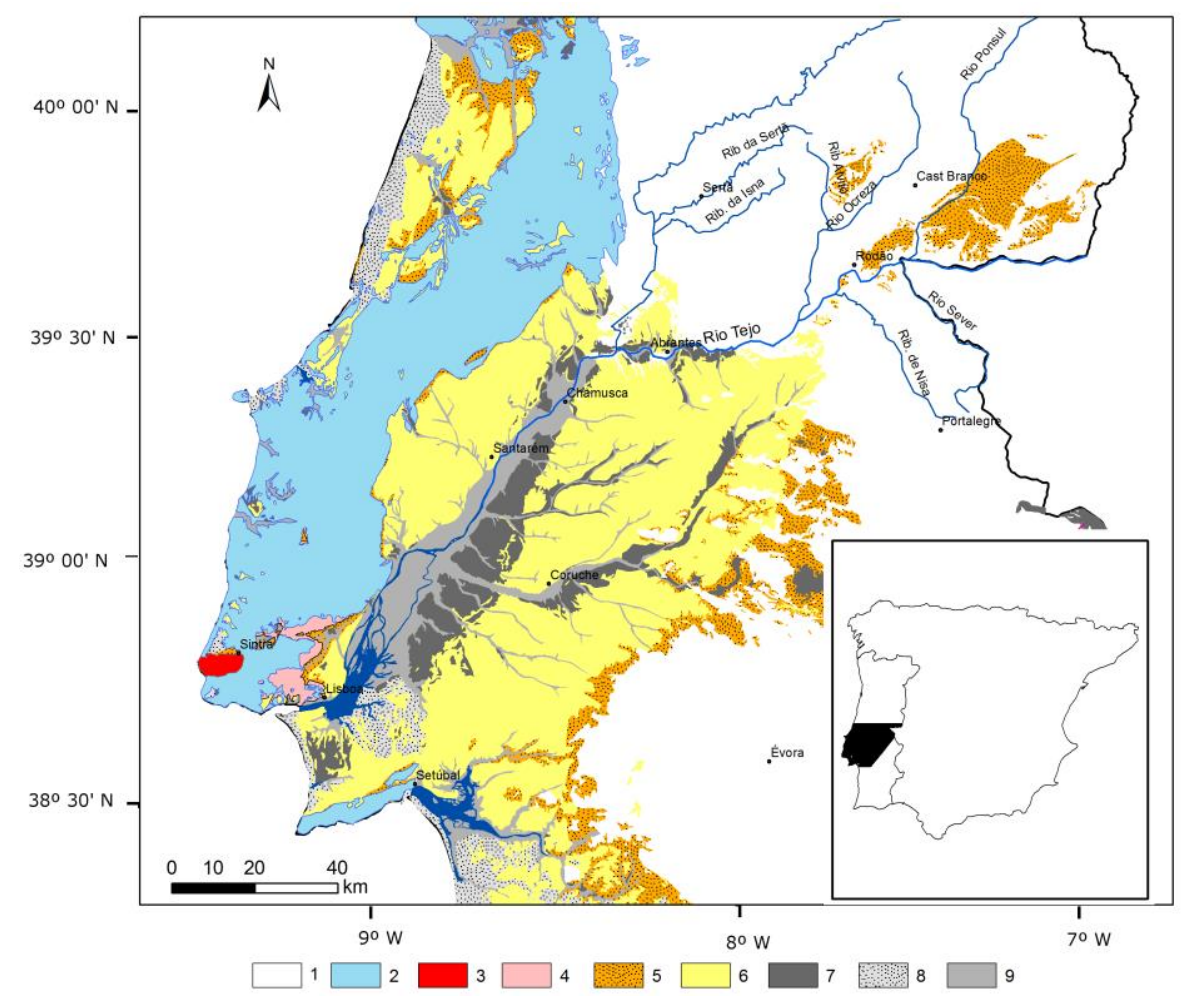

Fig. 1 - Mapa geológico da Bacia Cenozóica do Baixo Tejo e unidades morfo-estruturais envolventes (adaptado da Carta Geológica de Portugal 1: 500000, LNEG, 1992). 1 - Maciço Hespérico (Neoproterozóico e Paleozóico); 2 formações sedimentares mesozoicas; 3 - Maciço subvulcânico de Sintra (Cretácico); 4 - Complexo Vulcânico de Lisboa (Cretácico); 5 - conglomerados e arenitos (Paleogénico); 6 - arenitos, argilas e conglomerados (Neogénico); 7 - terraços fluviais cascalhentos (Plistocénico Inferior e Médio), 8 - terraços arenosos e areias eólicas (Plistocénico Superior), 9 - Aluviões (Holocénico).

Neste trabalho pretende-se apresentar uma síntese sobre a escadaria de terraços do Tejo dentro da bacia sedimentar cenozóica e sua relação com os leitos rochosos dos tributários situados em áreas a montante. 


\section{2 - Metodologia}

Existe uma extensiva literatura que descreve a forma dos perfis longitudinais dos rios regularizados. Todos eles podem ser descritos através de equações matemáticas: relação semi-logarítmica entre a distância para jusante e a altitude do canal (Hack, 1957, 1973); ou segundo uma relação potencial negativa entre o gradiente e a área a montante do troço (Wobus et al., 2006), ou igual relação entre o gradiente e o comprimento do canal desde a origem (Goldrick \& Bishop, 2007). Com o objectivo de relacionar os troços relíquia regularizados, nas cabeceiras dos tributários do Tejo, com as unidades morfoestratigráfica do Tejo, estudadas noutros trabalhos (Martins et al., 2009; Cunha et al., 2012), utilizou-se a equação do perfil de equilíbrio (Goldrick \& Bishop, 2007), equação 2. Onde: $\mathrm{H}=$ altitude do perfil de equilíbrio; $\mathrm{H}_{0}=$ uma estimativa da altitude teórica da divisória de águas, admitindo que os processos hidráulicos são activos até às cabeceiras de drenagem; $\mathrm{L}=$ comprimento do troço do rio desde a origem ao local considerado; $\mathrm{k}$ e $\lambda$ são constantes. A equação do perfil de equilíbrio é derivada da função potencial negativa que descreve a variação do gradiente com a distância a partir da origem para jusante nos perfis regularizados (equação 1).

$$
\begin{aligned}
& S=k \cdot L^{-\lambda} \\
& H=H_{0}-k \frac{L^{(1-\lambda)}}{1-\lambda}
\end{aligned}
$$

\section{3 - Resultados e discussão}

A projeção do perfil relíquia para jusante até à desembocadura dos tributários, permitiu calcular os valores da incisão fluvial desde a formação do terraço mais antigo do Tejo (T1), com cerca de 1 Ma (Tabelas 1 e 2). Embora os valores da incisão dos rios que correm na CCP não possam ser directamente comparáveis com os valores da incisão dos cursos de água que correm na superfície da Meseta Ibérica, porque as projeções para jusante usaram diferentes rios principais (o Zêzere na CCP e o Tejo para os rios da Meseta), dentro de cada grupo de tributários, os valores da incisão são mais elevados nos compartimentos sujeitos a maior soerguimento. É o caso da ribeira da Isna $(383 \mathrm{~m})$ por comparação com à ribeira da Sertã (149 m), que corre num compartimento mais deprimido, ou do rio Ocreza (214 m) e da ribeira de Nisa (209 m) por comparação com a ribeira da Pracana (116 m).

Apesar da escassez de dados geocronológicos, os perfis relíquia podem ser correlacionados com outras referências geomorfológicas existentes na região. A superfície fluvial culminante (SFC) da bacia do Baixo Tejo é considerada de idade Placenciana a Gelasiano (3,7 a 1,8 Ma), (Cunha 1992, 1996; Cunha et al., 


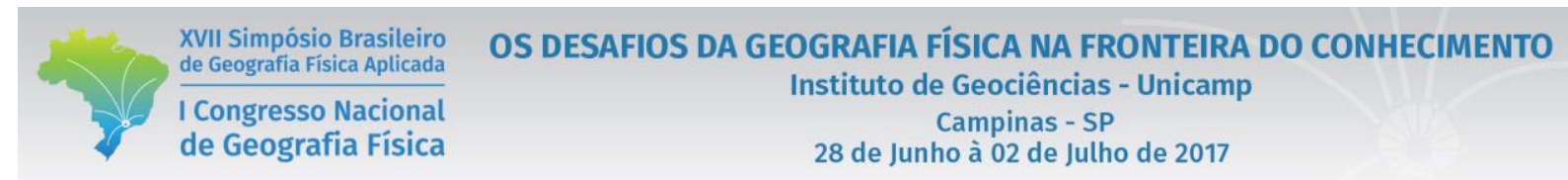

1993, 2012; Pais et al., 2012; Diniz et al., 2016) e os perfis relíquia, ligeiramente embutidos na superfície culminante, devem correlacionar-se com o terraço mais antigo do Tejo (T1), porque se encontram imediatamente abaixo daquela superfície e porque a sua projeção na desembocadura dos tributários se encontra a uma altura acima do leito actual do Tejo, similar à do terraço T1. Este terraço foi datado com cerca de 0,9 a 1Ma (Rosina et al., 2014).

Tabela 1 - valores de incisão calculados para os tributários do Tejo no interior da Cordilheira Central Portuguesa. $\mathrm{H}_{\mathrm{i}}$ = altitude inicial do troço analisado; $\mathrm{H}_{\mathrm{f}}=$ altitude final do troço analisado (Martins et al., 2017)

\begin{tabular}{c|cccccc}
\multicolumn{1}{c}{ Curso de água } & $\mathbf{H}_{\mathbf{0}}$ & $\mathbf{H}_{\mathbf{i}}$ & $\mathbf{H}_{\mathbf{f}}$ & $\mathbf{k}$ & $\boldsymbol{\lambda}$ & Incis $\tilde{\boldsymbol{a}} \boldsymbol{o}$ \\
\hline Sertã & 836 & 780 & 460 & 3.77 & 0.64 & $149 \pm 5$ \\
Isna & -132 & 900 & 670 & 166.9 & 1.10 & $383 \pm 12$ \\
Tamolha & 317 & 810 & 660 & 7093 & 1.45 & $283 \pm 2$
\end{tabular}

Tabela 2 - valores de incisão calculados para os tributários do Tejo na superfície da Meseta Ibérica. $\mathrm{H}_{\mathrm{i}}=$ altitude inicial do troço analisado; $\mathrm{H}_{\mathrm{f}}=$ altitude final do troço analisado (Martins et al., 2017)

\begin{tabular}{c|cccccc}
\multicolumn{1}{c}{ Curso de água } & $\mathbf{H}_{\mathbf{0}}$ & $\mathbf{H}_{\mathbf{i}}$ & $\mathbf{H}_{\mathbf{f}}$ & $\mathbf{k}$ & $\boldsymbol{\lambda}$ & Incis $\mathbf{A} \mathbf{O}$ \\
\hline Ocreza & 240 & 630 & 300 & 3612822 & 2.057 & $214 \pm 23$ \\
Sever & 699 & 600 & 490 & 1.512 & 0.563 & $199 \pm 2$ \\
\hline Ponsul/Alpreade & 5226 & 600 & 330 & 60.91 & 0.985 & $153 \pm 12$ \\
Nisa & 89 & 600 & 390 & 693 & 1.244 & $209 \pm 8$ \\
\hline Figueiró & 378 & 350 & 310 & 1.376 & 0.685 & $221 \pm 3$ \\
\hline Freixiada/Pracana & 746 & 560 & 410 & 15.47 & 0.855 & $186 \pm 7$ \\
\hline Pracana & 806 & 450 & 210 & 21.52 & 0.918 & $116 \pm 3$ \\
Canas & 839 & 300 & 220 & 24.55 & 0.933 & $105 \pm 3$ \\
Alpalhão & 362 & 340 & 290 & 1.44 & 0.580 & $159 \pm 3$
\end{tabular}

Uma das características geomorfológicas mais marcantes dos terraços do Baixo Tejo diz respeito ao grande desenvolvimento do terraço T1 e da sua correspondente lateral superfície de erosão (N1); o mesmo não acontece com os terraços médios e baixos (T3, T4, T5 e T6), confinados a um vale mais estreito e encaixado. Este facto configura um paradigma semelhante ao de vários rios europeus, cujos terraços mais antigos apresentam extensas superfícies agradacionais em oposição aos terraços inferiores, desenvolvidos 
em vales muito encaixados (ex. Bridgland \& Westaway, 2008; Westaway et al., 2009). O terraço T1 é anterior à "revolução do Plistocénico Médio" (Berger \& Jansen, 1994), ou seja, a sua formação ocupa vários ciclos de $41 \mathrm{ka}$, da ciclicidade climática de Milankovitch, enquanto as idades dos terraços médios e baixos parecem seguir razoavelmente o ritmo orbital da ciclicidade de 100 ka: T3 - MIS 11; T4 - MIS 9 a MIS 6; T5 - MIS 5; T6 - MIS 3 (Cunha et al., 2008; Martins et al., 2010a, 2010b; Cunha et al., 2016a, 2016b). No Plistocénico inicial dominaram descidas glácio-eustáticas do nível do mar, atingindo $70 \mathrm{~m}$. Durante a "Revolução do Plistocénico Médio" (1,2 Ma a 500 ka), progressivamente ocorreram descidas maiores (120 m) e com ciclicidade de $100 \mathrm{ka}$ (Gibbard \& Lewin, 2009).

Outro aspecto interessante diz respeito ao longo tempo de formação do terraço T1 no rio principal, podendo ser ligeiramente inferior nos tributários devido á transgressão temporal dos terraços de rocha (recuo dos knickpoints). Mesmo assim, a duração deverá ter sido suficiente para que os perfis dos tributários tivessem atingido uma situação de equilíbrio dinâmico (steady state) e uma regularização quase perfeita (Martins et al., 2017). Em perfis normalizados dos tributários do Tejo, dos quais se apresenta um exemplo (Fig. 2A), o ponto de maior concavidade do perfil encontra-se apenas a cerca de $20 \%$ da origem do perfil, uma proximidade indicadora do grau de regularização atingido pelos perfis longitudinais dos tributários do Tejo, ao tempo da formação do terraço T1. Actualmente, os perfis regularizados nas cabeceiras daqueles tributários, ligeiramente embutidos na superfície geral da Meseta, constituem "formas relíquia", anteriores ao mais recente e vigoroso encaixe da drenagem que caracteriza o troço a jusante (Fig. 2B). Considera-se que o soerguimento regional será a principal causa da disposição em escadaria dos seis níveis de terraço.

Além dos terraços mais antigos se terem desenvolvido durante ciclos climáticos de fraca amplitude, na fase inicial do encaixe, o Tejo disporia também de carga abundante proveniente do desmantelamento da SFC, situação que se alterou logo que o leito do rio principal atingiu o duro substrato metamórfico/granitóide subjacente à cobertura sedimentar, situação que ocorreu durante a incisão do terraço T2 e alargamento do strath do terraço T3.
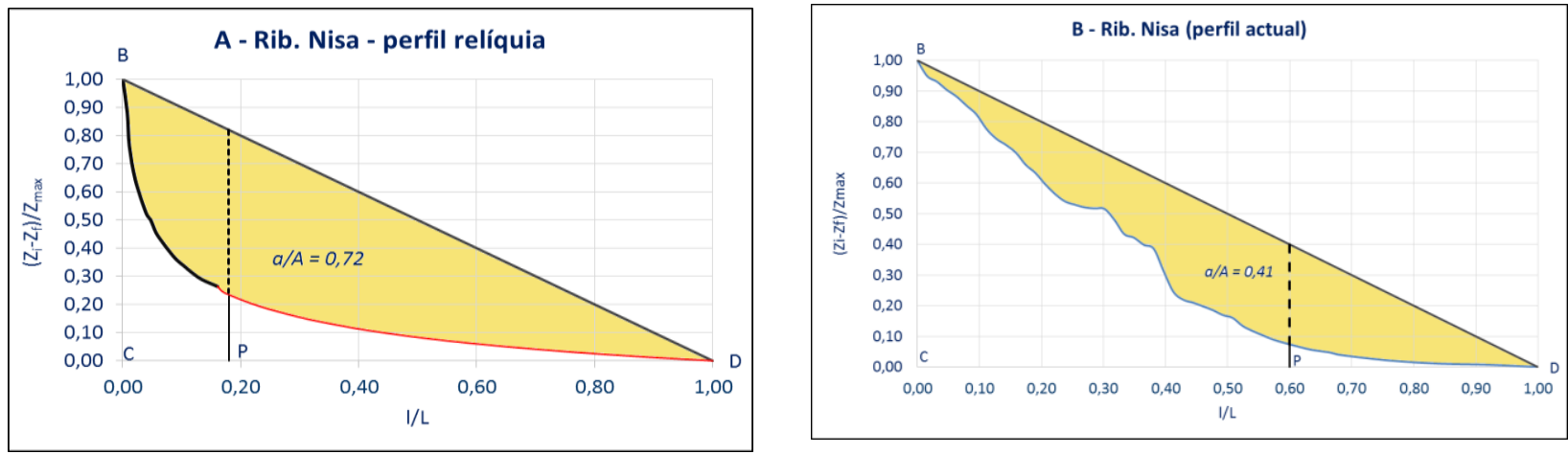
Fig. 2 (A) - Perfil relíquia normalizado da ribeira de Nisa (Portugal central oriental). Troço relíquia (linha a preto grossa) e sua projeção até à desembocadura (linha mais fina a vermelho). B = nascente do rio, $\mathrm{D}=$ desembocadura do rio, $a=$ área do polígono sombreado expressa em \% do triângulo $\mathrm{BCD}, \mathrm{A}=$ área do triângulo $\mathrm{BCD}, \mathrm{P}=$ ponto de maior concavidade expresso em percentagem de CD. A combinação das duas métricas $a / A$ e a posição do ponto de maior concavidade $(\mathrm{P})$, próximo da origem, claramente definem uma concavidade elevada $(a / A=0,72)$ do perfil longitudinal da ribeira de Nisa, ao tempo da formação do terraço T1. (B) - Perfil normalizado actual da ribeira de Nisa. A concavidade $(a / A=0,41)$, inferior ao do troço relíquia, claramente resulta do rejuvenescimento da drenagem, com ajustamento do perfil longitudinal a um nível de base mais baixo.

Os terraços médios e baixos, não dispondo de uma fonte de sedimento tão abundante e tendo-se desenvolvidos em ciclos climáticos de maior amplitude, as suas fases de incisão e agradação terão sido controladas pelas variações do nível de base de natureza glácio-eustáticas, comandadas pela ciclicidade orbital de cerca de $100 \mathrm{ka}$.

\section{4 - Conclusões}

Na Bacia Cenozóica do Baixo Tejo confirma-se a existência de seis níveis de terraços fluviais (T1 a T6), escalonados abaixo da unidade culminante do enchimento sedimentar (SLD13) e acima da respetiva planície aluvial.

Ciclos climáticos de $41 \mathrm{ka}$, relacionados com a obliquidade orbital durante o Plistocénico inicial, caracterizados pela fraca amplitude de variação do nível do mar, poderão explicar o longo tempo de formação do terraço T1 e a formação de perfis em equilíbrio dinâmico (steady state) à escala regional. Os terraços médios e baixos ter-se-ão formado já dentro da ciclicidade climática do Plistocénico Médio e Final (ciclos de $100 \mathrm{ka}$ ), que terão conduzido a fases de incisão mais pronunciadas, mas curtas, em comparação com as fases de agradação muito mais longas e coincidentes com posições mais altas do nível do mar.

O moderado soerguimento regional, que se intensifica no Plistocénico médio e final explica a disposição em escadaria dos diferentes níveis de terraço, enquanto o soerguimento diferencial terá condicionado os valores da incisão entre cursos de água com percurso na superfície da Meseta Ibérica e na CCP.

\section{Bibliografia}

BERGER, W. H.; JANSEN, E. Mid-Pleistocene climate shift: the Nansen connection. In: Johannessen, et al. (Ed.), The Polar Oceans and Their Role in Shaping the Global Environment. AGU Geophysical Monograph, vol. 85, 1994. p. 295-311.

BRIDGLAND, D. R.; WESTAWAY, R. Climatically controlled river terrace staircases: a worldwide Quaternary phenomenon. Geomorphology 98, 2008. p. 285-315. 
CABRAL, J. Neotectonics of mainland Portugal: state of the art and future perspectives. Journal of Iberian Geology 38 (1), 2012. p. 71-84.

CUNHA, P. P. Estratigrafia e sedimentologia dos depósitos do Cretácico Superior e Terciário de Portugal Central, a leste de Coimbra. 1992. 262 p. PhD Thesis Universidade de Coimbra, Portugal.

CUNHA, P. P. Unidades litostratigráficas do Terciário da Beira Baixa (Portugal). Comum. Instituto Geol Mineiro 82, 1996. p 87-130.

CUNHA, P. P.; ALMEIDA, N.; AUBRY, T.; MARTINS, A. A.; MURRAY, A. S.; BUYLAERT, J.-P.; SOHBATI, R., RAPOSO, L.; ROCHA, L. Records of human occupation from Pleistocene river terrace and aeolian sediments in the Arneiro depression (Lower Tejo River, central eastern Portugal). Geomorphology 165-166, 2012. p. 78-90.

CUNHA, P. P.; BARBOSA, B. P.; PENA DOS REIS, R. Synthesis of the Piacenzian on shore record, between the Aveiro and Setúbal parallels (Western Portuguese margin). Ciências da Terra,12, 1993. p. 35 - 43. Univ. Nova de Lisboa.

CUNHA, P. P; MARTINS, A. A.; BUYLAERT, J - P.; MURRAY, A.; RAPOSO, L.; MOZZI, P.; STOKES, M. New data on the chronology of the Vale do Forno sedimentary sequence (Lower Tejo River terrace staircase) and its relevance as a fluvial archive of the Middle Pleistocene in western Iberia. Quaternary Science Reviews, 2016b. http://doi.org/10.1016/j.quascirev.2016.11.001

CUNHA, P. P.; MARTINS, A. A.; GOUVEIA, M. P. As escadarias de terraços do Ródão à Chamusca (Baixo Tejo) - caracterização e interpretação de dados sedimentares, tectónicos, climáticos e do Paleolítico. Estudos do Quaternário, vol. 14, p. 1-24, 2016a. http://www.apeq.pt/ojs/index.php/apeq.

CUNHA, P. P.; MARTINS, A. A.; HUOT, S.; MURRAY, A.; RAPOSO, L. Dating the Tejo river lower terraces in the Ródão area (Portugal) to assess the role of tectonics and uplift. Geomorphology 102, 2008. p. 43-54.

DINIZ, F.; SILVA, C. M.; CACHÃO, M. O Pliocénico de Pombal (Bacia do Mondego, Portugal Oeste): biostratigrafia, paleoecologia e paleobiogeografia. Estudos do Quaternário/QuaternaryStudies 14, 2016. p. 41-59.

GIBBARD, P. L.; LEWIN J. River incision and terrace formation in the Late Cenozoic of Europe. Tectonophysics, 474, 2009. p. $41-55$.

GOLDRICK, G.; BISHOP, P. Regional analysis of bedrock stream long profiles: evaluation of Hack's SL form, and formulation and assessment of an alternative (the DS form). Earth Surf. Process. Landf. 32, 2007. p 649 671.

HACK, J. Studies of Longitudinal Stream Profiles in Virginia and Maryland. US Geol. Surv. Profess. Paper 294-B, 1957. p. $45-97$.

HACK, J. Stream profile analysis and stream-gradient index. J. Res. U. S. Geol. Surv. 1, 1973. p 421 - 429.

MARTINS, A. A.; CABRAL, J.; CUNHA, P. P.; STOKES, M.; BORGES, J.; CALDEIRA, B.; MARTINS, A. C. Tectonic and lithological controls on fluvial landscape development in central eastern Portugal: insights from long profile tributary stream analyses. Geomorphology 276, 2017. p. 144-163.

MARTINS, A. A.; CUNHA, P. P.; BUYLAERT, J.-P.; HUOT, S; MURRAY, A. S.; DINIS, P.; STOKES, M. KFeldspar IRSL dating of a Pleistocene river terrace staircase sequence of the Lower Tejo River (Portugal, western Iberia). Quaternary Geochronology 5 (2-3), 2010a. p. 176-180.

MARTINS, A. A.; CUNHA, P. P.; HUOT, S.; MURRAY, A.; BUYLAERT, J.-P. Geomorphological correlation of the tectonically displaced Tejo river terraces (Gavião-Chamusca area, Portugal) supported by luminescence dating. Quaternary International 199, 2009. p. 75-91.

MARTINS, A. A.; CUNHA, P. P.; ROSINA, P.; OOSTERBECK, L.; CURA, S.; GRIMALDI, S.; GOMES, J.; BUYLAERT, J.-P.; MURRAY, A. S.; MATOS, J. Geoarchaeology of Pleistocene openair sites in the Vila Nova da Barquinha - Santa Cita area (Lower Tejo River basin, central Portugal). Proceedings of the Geologists' Association 121 (2), 2010b. p. 128-140. 
PAIS, J.; CUNHA, P. P.; PEREIRA, D.; LEGOINHA, P.; DIAS, R.; MOURA, D.; BRUM DA SILVEIRA, A.; KullberG, J. C.; GONZÁleZ-DElGADO, J. A. The Paleogene and Neogene of Western Iberia (Portugal). A Cenozoic record in the European Atlantic domain. Springer Briefs in Earth Sciences.1vol. Springer Series ID: 8897, 2012. $158 \mathrm{p}$.

ROSINA, P.; VOINCHET, P.; BAHAIN, J.; CRISTOVÃO, J.; FALGUÈRES, C. Dating the onset of Lower Tagus River terrace formation using electron spin resonance. Journal of Quaternary Science 29 (2), 2014. p. 153-162.

WESTAWAY, R.; BRIDGLAND, D.; SINHA, R.; DEMIR, T. Fluvial sequences as evidence for landscape and climatic evolution in the Late Cenozoic: A synthesis of data from IGCP 518. Global Planetary Change, $68,2009$. p. 237-253.

WOBUS, C.; WHIPPLE, K.; KIRBY, E.; SNYDER, N.; JOHNSON, J.; SPYROPOLOU, K.; CROSBY, B.; SHEEHAN, D. Tectonics from topography: procedures, promise, and pitfalls. In: Willett, S., Hovius, N., Brandon, M., Fisher, D. (Eds.), Tectonics, Climate, and Landscape Evolution, Geol. Soc. Am. Spec. Pap., vol. 398, 2006. p. $55-74$. 